Revista de la Escuela de Ciencias de la Educación, año 15, nRo. 14, vol. 2, Julio a Diciemre de 2019. Páginas 1-13. ISSN 1851-6297 (DESDE DICIEMBRE DE 2006 A DICIEMBRE DE 2017). ISSN 2362-3349 (EN LÍNEA). LA INCORPORACIÓN DE LAS TIC EN LAS ESTRATEGIAS DIDÁCTICAS:

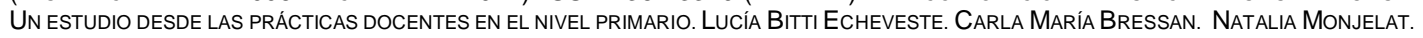

\title{
LA INCORPORACIÓN DE LAS TIC EN LAS ESTRATEGIAS DIDÁCTICAS: UN ESTUDIO DESDE LAS PRÁCTICAS DOCENTES EN EL NIVEL PRIMARIO
}

\author{
Lucía Bitti Echeveste* \\ Universidad del Gran Rosario, Argentina \\ lulibitti@gmail.com \\ Carla María Bressan* \\ Universidad del Gran Rosario, Argentina \\ carlabressan94@gmail.com \\ Natalia Monjelat* \\ CONICET - IRICE, Argentina \\ monjelat@irice-conicet.gov.ar
}

Recibido: 30/10/2018 Aceptado: 6/03/2019

\section{Resumen}

Este artículo presenta resultados de una investigación cualitativa, exploratoria y descriptiva, sobre la incorporación de las Tecnologías de la Información y la Comunicación (TIC) en las estrategias didácticas de docentes del nivel primario (segundo ciclo). La misma se realizó en dos escuelas de la ciudad de Rosario (Santa Fe, Argentina) empleando cuestionarios y entrevistas semi-estructuradas. A partir de un análisis estadístico descriptivo, se construyeron categorías de análisis emergentes y se identificaron respuestas textuales significativas. Considerando los discursos de los educadores sobre sus prácticas, los resultados indican que la totalidad de la muestra estaría incluyendo las TIC en sus estrategias didácticas, mostrando una diversidad en cuanto a la caracterización de los docentes, la utilización de diversos recursos y la implementación de éstos en diferentes actividades. Esto permitió identificar componentes y características de las estrategias didácticas tecnológicas en relación a la planificación educativa, las creencias de los docentes sobre estas herramientas, los criterios que establecen a la hora de seleccionar los recursos, la infraestructura tecnológica disponible y la capacitación en el uso de TIC. De esta forma, se aporta al conocimiento sobre el uso de TIC en el nivel primario, con datos concretos que permiten una exploración y descripción de estrategias didácticas en contextos situados.

\footnotetext{
* Estudiante de Licenciatura en Psicopedagogía (IUGR), con tesina aprobada y a la espera de fecha de defensa. Estudiante de profesorado de Nivel Inicial. Actualmente se desempeña como auxiliar de docente en Nivel Primario en un Centro Educativo de la ciudad de Rosario. Certificado de Educación Polimodal, modalidad Ciencias Naturales.

* Estudiante de Licenciatura en Psicopedagogía (IUGR), con tesina aprobada y a la espera de fecha de defensa. Estudiante de profesorado de Nivel Inicial. Actualmente se desempeña como auxiliar de docente en Nivel Primario en un Centro Educativo de la ciudad de Rosario. Certificado de Educación Polimodal, modalidad Ciencias Naturales.

* Doctora en Educación y Comunicación y Máster universitario en Comunicación y aprendizaje en la Sociedad Digital, títulos otorgados por la Universidad de Alcalá (UAH. Madrid, España). Licenciada en Psicopedagogía por la Universidad Nacional de San Martín (UNSAM, Argentina) y Diplomada en Constructivismo y Educación (FLACSO). Actualmente es miembro de la Carrera de investigador del Consejo Nacional de Investigaciones Científicas y Técnicas (CONICET) desempeñándose como Investigadora Asistente en el Instituto Rosario de Investigaciones en Ciencias de la Educación (IRICE: CONICET-UNR).
} 
Revista de la Escuela de Ciencias de la Educación, año 15, nRo. 14, vol. 2, Julio a Diciemre de 2019. PÁGinas 1-13. ISSN 1851-6297 (DESDE DICIEMBRE DE 2006 A DICIEMBRE DE 2017). ISSN 2362-3349 (EN LíNEA). LA INCORPORACIÓN DE LAS TIC EN LAS ESTRATEGIAS DIDÁCTICAS:

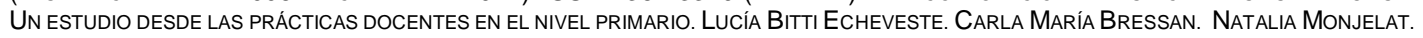

Palabras clave: TIC - Estrategias didácticas - Docentes - Investigación cualitativa - Nivel primario.

\begin{abstract}
This article presents results from a qualitative, exploratory and descriptive research on the incorporation of Information and Communication Technologies (ICTs) within the didactic strategies of elementary teachers. Data have been collected in two schools in the city of Rosario (Santa Fe, Argentina) employing questionnaires and semi-structured interviews. Analysis involved a descriptive statistical analysis that allowed the construction of emerging categories and the identification of significant textual responses. Considering the educators' discourses about their practices, the results indicate that the entire sample include ICT in their didactic strategies, showing diversity in the characterization of teachers, the use of resources and their implementation in different activities. This allowed the identification of components and characteristics of technological didactic strategies in relation to educational planning, teachers' beliefs about these tools, criteria to select resources, the access to technological infrastructure and ICT training. Therefore, the study contributes to the knowledge about the use of ICT at elementary education, with concrete data that allowed a situated exploration and description of didactic strategies.
\end{abstract}

\title{
Keywords: ICT- Didactic strategies- Teachers - Qualitative research - Elementary Education.
}

\section{Introducción}

Actualmente, existe consenso en torno a la idea que las tecnologías de la información y la comunicación (TIC) ocupan un lugar destacado en múltiples iniciativas curriculares, planes educativos y estrategias didácticas. En este sentido, la preocupación por la incorporación o integración de las TIC en los contextos educativos ha generado diferentes líneas de investigación, enfoques y posicionamientos que abordan, explican o sugieren diversas maneras de utilizar estos recursos en las aulas. Asimismo, considerando que los puntos de partida y las realidades territoriales de las instituciones suelen ser heterogéneas y desiguales (Tedesco, Steinberg y Tófalo, 2015), el estudio de esta problemática en contexto, desde estrategias cualitativas y casos, aparece como una propuesta destacada, que complementa las investigaciones que privilegian la representatividad a nivel nacional (Tófalo, 2015).

En este marco, la presente investigación exploratoria-descriptiva, tiene por objetivo estudiar la incorporación de las TIC dentro de las estrategias didácticas de docentes del nivel primario, identificando y describiendo las mismas en contextos específicos. Con ello se busca aportar conocimiento sobre los diferentes modos en que las TIC están siendo empleadas en las prácticas docentes, tomando para ello el caso de escuelas con alta disponibilidad tecnológica, ya que la presencia de tecnología es un requisito indispensable para la integración tecnológica, aunque como veremos luego, no es el único.

A continuación, se presentan los ejes conceptuales que guiaron el trabajo, para luego señalar la metodología empleada y los resultados obtenidos. Finalmente, se presentan conclusiones derivadas del estudio.

\section{Tecnologías de la información y la comunicación en la educación}

Diversos autores coinciden en señalar que las "nuevas tecnologías" generaron cambios en las formas de vinculación e interacción en la sociedad, siendo la palanca principal de transformaciones sin precedentes en el mundo contemporáneo, modificando significativamente los modos de comunicar, de entretener, de trabajar, de negociar, de gobernar y de socializar a escala global (Carneiro, 2006; Di Paolo, 2014; Dussel y Quevedo, 2010).

Garcés-Prettel, Ruiz-Cantillo y Martínez Ávila (2014) sostienen que en un escenario educativo que pretenda transformar la práctica pedagógica convencional, el disponer de infraestructura tecnológica y recursos informáticos es imprescindible. No obstante, aunque los recursos tecnológicos juegan un papel importante, la trascendencia dentro del proyecto educativo tiene que ver con las formas innovadoras de usar o integrar las tecnologías para fortalecer el aprendizaje, brindando a los estudiantes nuevas posibilidades educativas en las distintas áreas del conocimiento o del currículo en general, en donde los sujetos más que compartir conocimientos e información, compartan significados, representaciones, valores, actividades y usos tecnológicos que despierten el deseo por aprender. En relación a esto, algunos autores sostienen que integrar pedagógicamente a las TIC no implica hacer foco exclusivo en el uso de equipamientos y herramientas que ingresan a las instituciones educativas por un imperativo externo. Más bien, consideran que las tecnologías ingresan a las instituciones como una oportunidad para lograr un cambio en las prácticas docentes y, a su vez, para que los estudiantes alcancen aprendizajes más significativos. La adecuación a los contextos, la posibilidad de respuesta a necesidades y el sentido que logren adquirir en torno a proyectos individuales y colectivos parecen ser algunas de las claves para una integración efectiva (Batista, 2007; Lugo y Kelly, 2011). 
En esta línea, Escontrela Mao y Stojanovic Casas (2004) hacen hincapié en que los usos educativos de las TIC no surgen de forma automática porque exista la posibilidad técnica, sino porque hay actores institucionales con proyectos educativos que aprovechan la potencia comunicativa de estas tecnologías para llevarlos a cabo. Asimismo, Cabello y Levis (2007) sostienen que las tecnologías educativas no son el fin ni los condicionantes de una buena práctica de enseñanza. Si se utilizan las TIC para reforzar nuevas formas innovadoras de aprendizaje y así crear nuevos entornos de aprendizajes en una escuela, el proceso no tiene que ver con éstas como tales, sino que el cambio está relacionado con el estilo de gestión, la actitud y la formación del profesorado, los enfoques pedagógicos y los nuevos estilos de aprendizaje (Cabello y Levis, 2007; Escontrela Mao y Stojanovic Casas, 2004). En este sentido, Coll (2009) plantea que no se trata de utilizar las TIC para hacer lo mismo, pero mejor, con mayor rapidez y comodidad o incluso con mayor eficacia. De manera contraria, se las usa para hacer cosas diferentes, para poner en marcha procesos de aprendizaje y de enseñanza que no serían posibles en ausencia de éstas.

Por su parte, García-Prettel y sus colegas (2014) señalan la existencia de diferentes maneras de integrar las TIC en el sistema educativo, siendo las más comunes: la integración para la gestión administrativa, la integración para la gestión curricular o pedagógica, la integración para el diseño e implementación de proyectos pedagógicos.

Cabello (2012) explica que se requieren procesos largos y complejos para que las tecnologías digitales puedan orientar las prácticas educativas, señalando que generalmente la integración es fragmentaria, difícil, poco competente y lenta. Las tensiones y contradicciones se manifiestan en distintas dimensiones del problema: la política educativa, el equipamiento, la capacitación de los docentes, entre otros. En la misma línea, Lerner (2012) también plantea que la incorporación de las nuevas tecnologías a la enseñanza no se logra mágicamente a partir de la llegada de las computadoras. Por el contrario, es un proceso que se desarrolla con el tiempo. La autora cuenta que algunos maestros están tan alejados de la computadora que no pueden imaginar para qué podría utilizarse en la escuela; otros están familiarizados sólo con algunos de sus usos y esto parece determinar una representación muy parcial de sus potencialidades para el aprendizaje; otros conocen bien tanto sus posibilidades como las dificultades que se plantean al utilizarla con los alumnos e intentan definir en qué condiciones es productiva su incorporación a la tarea.

En el contexto local, se relevan investigaciones que exploran diversos usos de diferentes tecnologías en contextos escolares (Canales, 2017; Salvucci Gaido, 2017; Tófalo, 2015, 2017). Adicionalmente, en el estudio de Guiller (2015) los docentes sostienen que se les brindan recursos tecnológicos, pero la capacitación se basa generalmente en sus propias experiencias en las instituciones donde trabajan. La diversidad de experiencias también es documentada por Cariaga (2018) en los diferentes niveles educativos, concluyendo que algunos profesores han instrumentado cambios favorables en sus prácticas, mientras otros revelan prácticas tradicionales en las que se usan las TIC sólo para apoyar viejos modos de enseñar.

\section{La presencia de las TIC en las estrategias didácticas}

Según diversos autores, es posible señalar que las estrategias didácticas comprenden un diagnóstico de la realidad, la selección de métodos didácticos, las tareas por realizar, la selección de actividades y prácticas pedagógicas en diferentes momentos, la planeación de los recursos y la forma de evaluación del proceso formativo (Bixio, 2005; Burgess y Russell 2003; Cid-Sabucedo, Pérez-Abellás y Zabalza; 2009; González y Ramírez, 2010; González Jaimes et al., 2015). De esta forma, se puede afirmar que son fundamentalmente procedimientos relacionados con los procesos de enseñanza y aprendizaje y que poseen una intencionalidad y motivaciones definidas. En la misma línea de pensamiento, Sánchez Rosal (2010) considera que al momento de diseñar o producir un material educativo y escoger la estrategia didáctica, es necesario establecer una planificación educativa fundamentada en alguna teoría de aprendizaje. Asimismo, existe una diversidad de estrategias didácticas, ya que éstas van a depender de la subjetividad del docente, los recursos existentes y del propio contexto donde se dan las acciones didácticas (Feo, 2010).

En cuanto a los recursos, en este trabajo nos interesan particularmente las estrategias didácticas donde se emplean herramientas tecnológicas digitales. Litwin (2005) acuña el concepto de "didáctica tecnológica" para explicar y pensar el uso concreto de las TIC en educación, resaltando el aspecto pedagógico por sobre lo tecnológico. Para la autora, la didáctica tecnológica se conforma por un cuerpo de conocimientos referidos a las prácticas de la enseñanza configuradas en relación con los fines que le dan sentido al acto de enseñar. Ese cuerpo de conocimientos reconoce, además, la influencia de las TIC en la educación y abarca las características de las estrategias docentes cuando son mediadas tecnológicamente. Considerando estas cuestiones, Roig (2016) plantea la necesidad de construir categorías teóricas que permitan mirar a la tecnología didácticamente, sosteniendo que las tecnologías "ya están", es por esto, que no podemos hablar de "introducirlas" en las aulas, sino que es necesario revisar cómo las percibimos y cómo las usamos. 
A partir de lo planteado, se puede visualizar que existen diferentes formas de uso significativo de las TIC en el contexto escolar, que pueden acarrear cambios en las formas de enseñar y de aprender de los distintos actores institucionales. Coll (2009) sostiene que la capacidad de transformación y mejora de la educación con las TIC debe entenderse como un potencial que puede o no hacerse realidad, dependiendo del contexto en el que las tecnologías sean efectivamente utilizadas. Es decir, son los contextos de uso y la finalidad que se persigue con la incorporación de las TIC, los que determinarán la posibilidad de transformar y mejorar los procesos de enseñanza y aprendizaje. Por esta razón, interesa conocer el estilo de enseñanza, el modo de presentar los contenidos, los objetivos y la intencionalidad educativa que persiguen los diferentes actores institucionales al incorporar las TIC, entendiendo a la escuela como un contexto educativo en el que la cultura actúa a través de éste como mediadora del desarrollo socio-cognitivo (Cole, 1984). Bixio (2005) sostiene que se trata de un contexto tanto físico, refiriendo al edificio institucional, como socio-cultural en el que tienen lugar determinadas prácticas socio-institucionales que "marcan" los procesos de enseñanza y aprendizaje y los conocimientos que allí se construyen.

Rivero Cárdenas, Gómez Zermeño y Abrego Tijerina (2013) identificaron que casi la totalidad de los docentes que conformaron su muestra, consideraban que sí es posible incorporar las TIC en el desarrollo de diferentes estrategias didácticas. A su vez, el $85 \%$ de los docentes, consideraron favorable la combinación de estrategias didácticas y las TIC para facilitar el aprendizaje a sus estudiantes. Por su parte, Area Moreira (2010) observó en una investigación española que las actividades planificadas con TIC suelen plantearse como un complemento de la materia/asignatura que se está trabajando.

Considerando estas cuestiones, resulta importante estudiar en profundidad las estrategias didácticas, entendidas como los modos de actuar por parte de los docentes que tienen como fin generar aprendizajes significativos en los estudiantes, a través de acciones y herramientas, en este caso de TIC, que buscan facilitar los procesos de enseñanza y aprendizaje en el contexto local.

\section{Marco metodológico}

Para el logro de los objetivos propuestos se ha llevado a cabo una investigación cualitativa. Se buscó comprender los fenómenos en estudio teniendo en cuenta la forma en que los participantes perciben subjetivamente su realidad, profundizando en sus experiencias, perspectivas, opiniones y significados (Hernández Sampieri, Fernández Collado y Baptista Lucio, 2014). Adicionalmente, se trata de una investigación exploratoria-descriptiva (Sabino, 1996) ya que las variables a estudiar, estrategias didácticas y $\mathrm{TIC}$, han sido poco exploradas y descriptas específicamente en contextos de alta disponibilidad tecnológica como suele ser el caso de las escuelas privadas en la ciudad de Rosario que componen la muestra, tal como se desarrolla en el siguiente apartado.

\section{Contexto y Participantes}

Siguiendo una metodología cualitativa, la muestra se seleccionó de manera no probabilística, buscando casos "típicos", sin pretender que sean estadísticamente representativos de una población determinada. Específicamente, se realizó un "muestreo cualitativo por conveniencia", accediendo a casos disponibles a los que se tenía posibilidad de analizar (Hernández Sampieri et al., 2014). Asimismo, la elección de la muestra se realizó sabiendo con anterioridad que las escuelas contaban con la infraestructura tecnológica necesaria para responder a nuestros objetivos de investigación. El trabajo de campo se desarrolló entonces en el espacio de dos escuelas pertenecientes a la ciudad de Rosario, a las que llamaremos "institución A" e "institución B". Ambas son de gestión privada y con una oferta educativa que abarca los tres niveles, Inicial, Primario y Secundario. Los estudiantes que a ellas asisten corresponden a un nivel socio-económico medio y medio-alto.

\section{Recolección y análisis de datos}

En una primera instancia se diseñó un cuestionario ad hoc semi-estructurado con preguntas abiertas y cerradas, para recolectar información sobre las opiniones, creencias y prácticas de los docentes de segundo ciclo relacionadas con el problema de estudio. Asimismo, se buscó obtener información demográfica de la muestra a través de preguntas que indagaban la edad, profesión, asignatura y curso a cargo de cada una de las encuestadas. Así pues, el mismo nos permitió indagar sobre las estrategias didácticas en general, para poder identificar dentro de la muestra a aquellos docentes que sí incluían TIC en sus prácticas. En caso de responder afirmativamente, se solicitó responder a su vez qué TIC utilizaban y utilizarían próximamente, así como también qué actividades realizaban y realizarían con las mismas en sus clases. En caso contrario, se buscaba saber qué otros recursos utilizaban las profesionales en sus estrategias didácticas. Este instrumento, al ser económico y rápido (Sabino, 1996), fue administrado a la totalidad de los docentes de segundo ciclo de ambas instituciones $(n=23)$.

En una segunda instancia y a partir de un análisis preliminar de los cuestionarios, se elaboró una entrevista semi-estructurada (Arnold, Spedding y Pereira, 2013) buscando profundizar en la relación 
Revista de la Escuela de Ciencias de la Educación, año 15, nRo. 14, vol. 2, Julio a Diciemre de 2019. Páginas 1-13. ISSN 1851-6297 (DESDE DICIEMBRE DE 2006 A DICIEMBRE DE 2017). ISSN 2362-3349 (EN LíNEA). LA INCORPORACIÓN DE LAS TIC EN LAS ESTRATEGIAS DIDÁCTICAS:

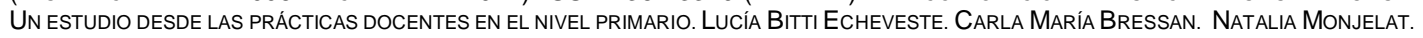

existente entre las estrategias didácticas y las tecnologías digitales. Para ello, se diseñaron preguntas que permitieran identificar las diferentes concepciones y maneras de incluir las TIC por parte de las maestras entrevistadas, consultar sobre la infraestructura tecnológica y los recursos informáticos disponibles en cada escuela, las diversas maneras de seleccionarlos y usarlos por parte de los profesionales, la capacitación docente en el uso de las TIC, los estilos de enseñanza, objetivos y planificación educativa que ponen en obra las docentes.

Para dicha selección nos basamos en diferentes criterios buscando diversidad: la edad de las profesionales, sus titulaciones, las asignaturas que tienen a cargo, los recursos y actividades tecnológicas que mencionaron en los cuestionarios, así como aquellas estrategias didácticas nombradas que no incluían TIC. Este instrumento se administró a 4 maestras, dos de cada institución, referenciadas como A6 y A9, B6 y B9.

En cuanto al procesamiento y análisis de los datos, se comenzó realizando un análisis estadístico descriptivo de las variables presentes en el cuestionario, lo que permitió caracterizar a la muestra y explorar preliminarmente cuestiones relacionadas al uso de TIC en las estrategias didácticas. Para las preguntas cerradas, se confeccionó una matriz de datos con cada una de éstas y, a partir de allí se realizó el análisis descriptivo, calculando los porcentajes para cada una de las respuestas y realizando distribuciones de frecuencias. En cuanto a las preguntas abiertas, se construyeron categorías de análisis emergentes luego de realizar una lectura inicial de los datos y considerando los aportes del marco teórico. A partir de esto se armó una matriz de datos, dando lugar a un análisis descriptivo tal como en las preguntas cerradas (Hernández Sampieri et al., 2014). Por otra parte, se identificaron respuestas textuales significativas que permitieran ejemplificar los datos relevados de manera cuantitativa.

Asimismo, se realizó un análisis cualitativo de las cuatro entrevistas, siguiendo lo sugerido por Hernández Sampieri et al. (2014). En primer lugar, se transcribieron las entrevistas utilizando un programa denominado O'Transcribe. Éstas dieron lugar a segmentos de contenidos que permitieron generar categorías de análisis emergentes basadas tanto en la revisión bibliográfica como en los datos previamente recolectados y analizados.

Algunas categorías permitieron ordenar los datos y generar luego nuevamente distribuciones de frecuencias, como en el caso por ejemplo del tipo de tecnología presente en cada institución escolar. Sin embargo, en otros casos, las categorías reflejan un esfuerzo por agrupar patrones identificados en los datos. En esta línea se destacan las categorías emergentes que se describen en la tabla 1, utilizadas en el análisis de las actividades que las docentes señalaron tanto en los cuestionarios como en las entrevistas.

Tabla 1. Descripción de las categorías de análisis

Visualización de videos:

Recreación educativa:

Uso de programas digitales:

Presentación o ampliación de un tema:

Uso de Internet:

Exposición de trabajos

por parte del alumnado:

Búsqueda de información:
Uso de algún recurso tecnológico que permite la visualización de videos.

Utilización de las tecnologías para escuchar música, sacar fotos, mirar películas, jugar a diferentes juegos con o sin un fin pedagógico o, simplemente, con el objetivo de generar un momento de dispersión dentro de los contenidos escolares.

Uso de tecnologías para realizar actividades implicando diferentes programas o softwares digitales. Se observa la presencia de programas generales y programas específicos de ciertas materias.

Implementación de recursos tecnológicos para presentar o ampliar contenidos en las clases.

Incorporación de las tecnologías para utilizar el navegador y otras páginas de Internet.

Uso de tecnologías para presentación de trabajos

Uso de las TIC para obtener información relacionada a los contenidos escolares. 
Revista de la Escuela de Ciencias de la Educación, año 15, nRo. 14, vol. 2, Julio a Diciemre de 2019. Páginas 1-13. ISSN 1851-6297 (DESDE DICIEMBRE DE 2006 A DICIEMBRE DE 2017). ISSN 2362-3349 (EN LÍNEA). LA INCORPORACIÓN DE LAS TIC EN LAS ESTRATEGIAS DIDÁCTICAS: Un estudio desde las prácticas docentes en el nivel primario. Lucía Bittı EcheVeste. Carla María Bressan. Natalia Monjelat.

El trabajo con otras materias:

Implementación de tecnologías que requiere el trabajo en conjunto de diferentes materias de la institución.

Actividades que impliquen opinar o debatir sobre algún tema:

Actividades con TIC que dan lugar a debates entre el alumnado.

Por último, en base a las categorías identificadas, se comenzó a vincular los resultados con el conocimiento disponible para entender el fenómeno de estudio, discutir los resultados y arribar a algunas conclusiones.

\section{Resultados}

Características de la muestra: un primer análisis de los datos

Como presenta la tabla 2, el análisis de los cuestionarios mostró que un $30 \%$ de las encuestadas tienen entre 46 y 50 años. En cuanto a la titulación, se observaron diferentes perfiles con una predominancia de docentes que realizaron un profesorado. A su vez, aunque algunas docentes dan clases en más de un curso, el $37 \%$ de la muestra corresponde a docentes de 6 to grado.

Tabla 2. Caracterización de la muestra.

\begin{tabular}{|c|c|c|c|}
\hline Variable & Categoría & Frecuencia & Porcentaje (\%) \\
\hline \multirow{8}{*}{ Edades } & $20-25$ & 3 & $13,04 \%$ \\
\hline & $26-30$ & 4 & $17,39 \%$ \\
\hline & $31-35$ & 1 & $4,34 \%$ \\
\hline & $36-40$ & 3 & $13,04 \%$ \\
\hline & $41-15$ & 3 & $13,04 \%$ \\
\hline & $46-50$ & 7 & $30,43 \%$ \\
\hline & $51-55$ & 2 & $8,69 \%$ \\
\hline & Total & 23 & $100 \%$ \\
\hline \multirow{5}{*}{ Cursos a cargo } & 4to grado & 8 & $22,85 \%$ \\
\hline & 5 to grado & 9 & $25,71 \%$ \\
\hline & 6 to grado & 13 & $37,14 \%$ \\
\hline & $7 \mathrm{mo}$ grado & 5 & $14,28 \%$ \\
\hline & Total & $35^{*}$ & $100 \%$ \\
\hline \multirow{7}{*}{ Titulaciones } & $\begin{array}{l}\text { Profesora de Educación } \\
\text { Primaria }\end{array}$ & 10 & $43,47 \%$ \\
\hline & $\begin{array}{l}\text { Ingeniera en sistemas } \\
\text { informáticos }\end{array}$ & 1 & $4,34 \%$ \\
\hline & Licenciada en Bellas Artes & 1 & $4,34 \%$ \\
\hline & Profesora de Inglés & 3 & $13,04 \%$ \\
\hline & Psicóloga & 1 & $4,34 \%$ \\
\hline & No responde & 7 & $30,43 \%$ \\
\hline & Total & 23 & $100 \%$ \\
\hline
\end{tabular}

${ }^{*}$ El total de docentes de la muestra es 23 , pero algunas de ellas dictan clases en diferentes cursos, por ende, el total en este caso es 35 .

Actividades de las docentes: recursos y creencias respecto a las estrategias didácticas con tecnologías

Una vez caracterizada la muestra, se procedió a analizar en los cuestionarios aquellas preguntas vinculadas particularmente con el uso de tecnologías en la práctica docente. Partimos de la premisa de que no todas emplearían estos recursos y por eso optamos por administrar el cuestionario inicial a todas 
Revista de la Escuela de Ciencias de la Educación, año 15, nRo. 14, vol. 2, Julio a Diciemre de 2019. Páginas 1-13. ISSN 1851-6297 (DESDE DICIEMBRE DE 2006 A DICIEMBRE DE 2017). ISSN 2362-3349 (EN LÍNEA). LA INCORPORACIÓN DE LAS TIC EN LAS ESTRATEGIAS DIDÁCTICAS:

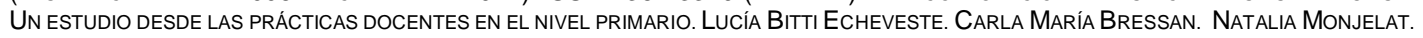

las docentes del nivel. Sin embargo, el análisis de los 23 cuestionarios reveló que el 100\% incluyen las TIC en sus prácticas.

En la figura 1, se resumen los recursos más utilizados por las profesionales. Se observa a su vez que 5 maestras emplean recursos no digitales tales como mapas, fotos, juegos, afiches, posters y manuales dentro de sus estrategias. Estos datos se complementaron con los obtenidos en las entrevistas, donde las docentes se explayaron respecto al uso de recursos tecnológicos en sus prácticas docentes, como parte de sus estrategias didácticas.

Los datos recolectados en las entrevistas confirmaron la disponibilidad tecnológica de cada institución. La institución A posee una TV Smart y una computadora en cada salón y algunos de éstos cuentan asimismo con un grabador. Las docentes de las materias especiales, para poder disponer de recursos como computadora/notebook, televisor o proyector, deben pedirlos con anterioridad. Las aulas asignadas a materias específicas, como mencionó la docente de Tecnología en su entrevista, están mejor equipadas que las de las materias especiales. En los discursos de las entrevistadas de la escuela A, se hizo presente el mal funcionamiento del Wi-fi, lo cual dificulta el uso de Internet llevando a las maestras a utilizar otros medios como el celular o el pen drive.

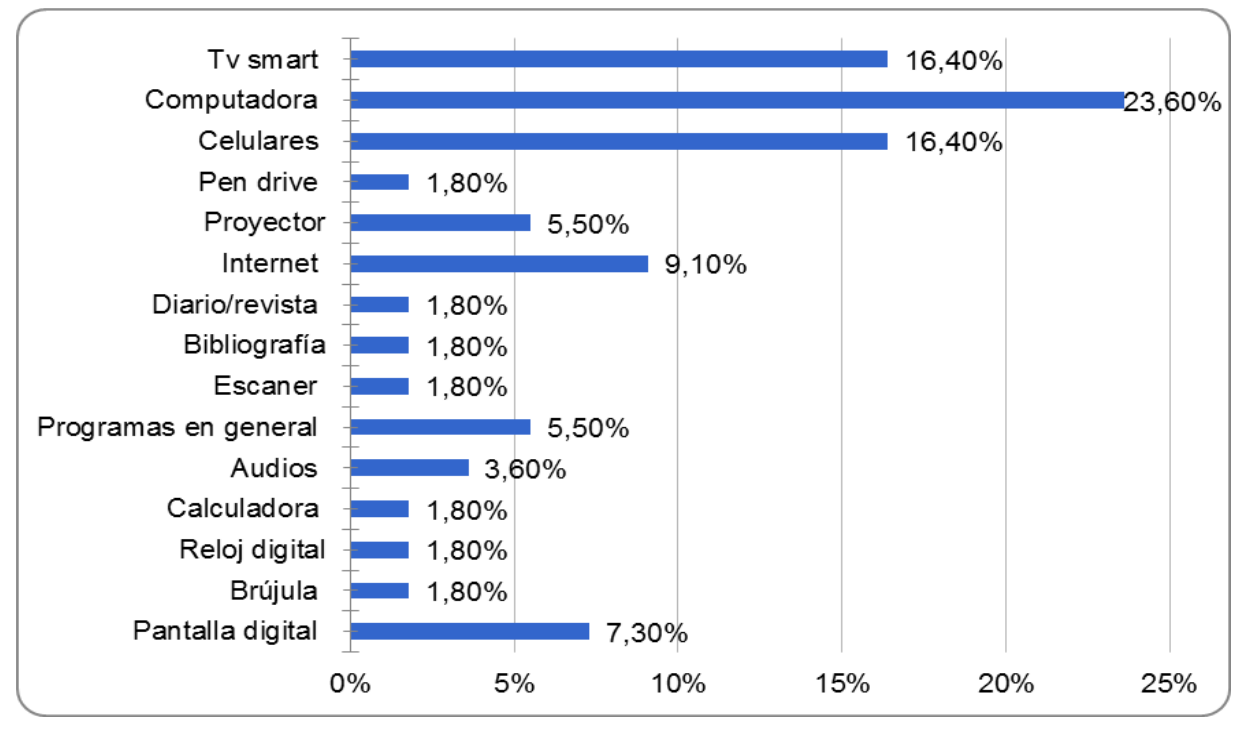

Figura 1. Tecnologías utilizadas por los docentes

Por su parte, la institución B cuenta con pizarras digitales interactivas con acceso a Internet, computadoras y micrófonos en cada salón. A su vez, tiene dos espacios disponibles: la sala de computación, donde cada estudiante puede disponer de una computadora para realizar actividades, y la sala de innovación, con un televisor, una pantalla blanca, diez computadoras, una notebook y un cañón. A partir de esta descripción, se infirió que en ambas escuelas la disponibilidad tecnológica es alta.

Otro aspecto que resulta interesante a la hora de estudiar las estrategias de las docentes, es qué concepciones o creencias tienen las mismas sobre las TIC, aspectos que abordamos en las entrevistas. Las cuatro maestras coincidieron en señalar que las TIC son una gran herramienta que ayudan a que se den los procesos de enseñanza y aprendizaje, mencionamos las palabras de dos entrevistadas: "son instrumentos las tecnologías - que te ayudan y facilitan, generan mayor interés por parte de los alumnos, es más entretenido, para ellos y para uno" (A6); "estoy abierta al uso de las TIC, creo que son necesarias porque los chicos que estamos teniendo son nativos digitales (...) están atravesados por la tecnología, y la escuela no puede quedar afuera" (A9). Las cuatro expresaron que las tecnologías son recursos que facilitan y mejoran la calidad de la enseñanza y la comprensión de un tema, lo cual lleva a que se genere un mayor interés en la clase por parte de los alumnos: B2 sostuvo que las TIC "son recursos que facilitan la enseñanza y sobre todo lo que facilitan es la comprensión de los temas que uno aborde", mientras que B9 expresó que para ella "son un recurso que utilizo a diario para mejorar la calidad de enseñanza con mis alumnas".

A su vez, los discursos docentes mostraron que una de las maneras que tienen los maestros de acompañar los procesos de enseñanza y aprendizaje, es utilizar a estas tecnologías como soporte o complemento para la explicación de un contenido:

(...) es cuestión de concientizarnos todos, en la responsabilidad del uso -de las tecnologías- y 
Revista de la Escuela de Ciencias de la Educación, año 15, nRo. 14, vol. 2, Julio a Diciemre de 2019. Páginas 1-13. ISSN 1851-6297 (DESDE DICIEMBRE DE 2006 A DICIEMBRE DE 2017). ISSN 2362-3349 (EN LíNEA). LA INCORPORACIÓN DE LAS TIC EN LAS ESTRATEGIAS DIDÁCTICAS:

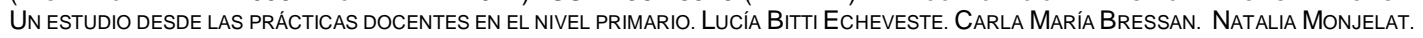

en la responsabilidad de su uso adecuado en la educación. Porque si impacta de manera negativa o reemplaza mi trabajo como docente, ni una cosa ni la otra. Me parece que se tienen que complementar las cosas, no de que les paso un vídeo y media hora de clase yo estoy en otra... o miramos una película porque el viernes estoy cansada. Si esa película tiene un fin didáctico o algo que sume, que sea una manera de aprendizaje, si... pero, si es para suplantar otra cosa, no es un uso razonable, adecuado. Pasa por ahí, me parece a mí, el uso de la tecnología (A9).

\section{Estrategias didácticas: sus componentes y características en relación a las TIC}

Por otra parte, nos interesaba conocer cómo son empleados los recursos tecnológicos disponibles en estas escuelas, para poder describir las características y componentes de las diferentes estrategias didácticas. Como decíamos previamente, contar con una infraestructura tecnológica no es el único factor a tener en cuenta, por lo que también se indagó a través de los cuestionarios y de las entrevistas sobre qué actividades realizaban las docentes con estos recursos.

Como se observa en la figura 2 , un $70 \%$ del total de docentes encuestadas, utiliza las TIC para la visualización de vídeos, recreación educativa, uso de programas en general y presentación de un tema; mientras que el $4 \%$ no respondió a esta pregunta.

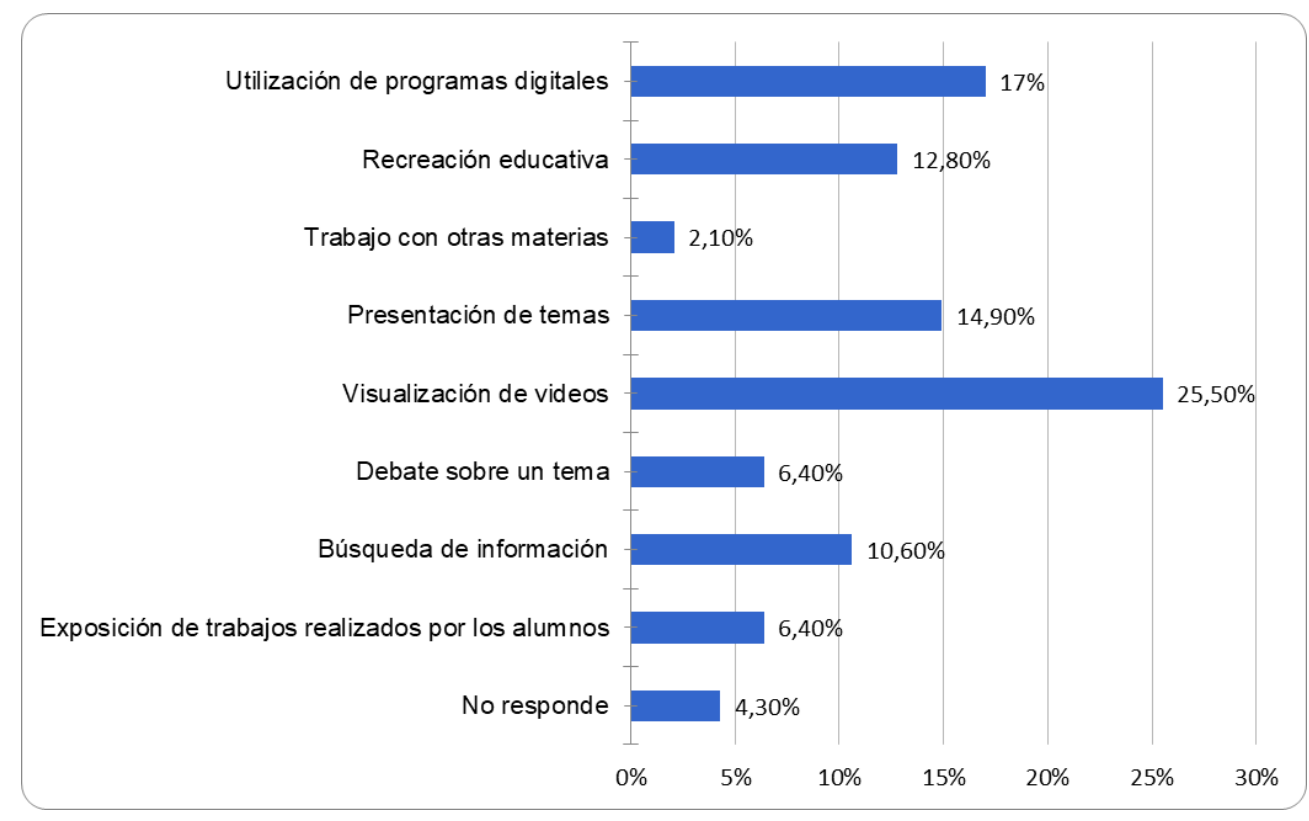

Figura 2. Actividades realizadas con TIC

El porcentaje restante de la muestra dio a conocer que incorpora las tecnologías para otras actividades como trabajar con otras materias, debatir sobre determinado tema, buscar información y dar lugar a que los estudiantes expongan trabajos. Estas actividades se presentaron a su vez en los discursos de las docentes entrevistadas, complementando los datos recogidos inicialmente en las preguntas abiertas de los cuestionarios.

Cabe señalar que en algunas respuestas se observa una combinación de actividades. Por ejemplo, ese es el caso de la docente A5, quien se refirió a la visualización de videos para la presentación de algún tema o para la explicitación del mismo: "Vimos videos sobre temas de Lengua, por ejemplo, mitos". A su vez, A7 mencionó que utiliza "Videos educativos, por ejemplo, para trabajar el tema biomas de Argentina". Específicamente, las cuatro entrevistadas coincidieron en que la utilización de vídeos ayuda, facilita, y genera un mayor interés en el alumnado, sosteniendo que éstos son recursos que generan aprendizajes más ricos y productivos, ya que, como menciona A6: "es un recurso mucho más positivo con respecto a la parte de imágenes visuales, sonidos, se puede trabajar mucho más con los sentidos...con otros sentidos que son más dinámicos".

Por otro lado, las docentes señalaron el uso de recursos tecnológicos durante las clases. Se observan diferentes programas informáticos y plataformas digitales que pueden utilizarse para diferentes fines y en diferentes asignaturas y niveles, tales como Power Point, YouTube, Word, Excel, Prezi, Portal Educ.ar. 
Revista de la Escuela de Ciencias de la Educación, año 15, nRo. 14, vol. 2, Julio a Diciemre de 2019. Páginas 1-13. ISSN 1851-6297 (DESDE DICIEMBRE DE 2006 A DICIEMBRE DE 2017). ISSN 2362-3349 (EN LíNEA). LA INCORPORACIÓN DE LAS TIC EN LAS ESTRATEGIAS DIDÁCTICAS:

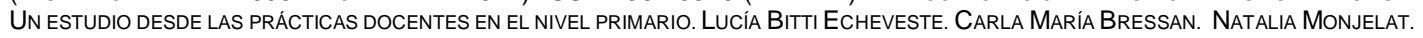

Asimismo, en las actividades se mencionan programas, entornos y plataformas que permiten trabajar contenidos particulares: Matepractic, Biblioteca virtual de libros en Inglés, Google maps, Google Earth, Geogebra, Matematicán, Bambi lector. Al respecto, la docente B3 expresó que utiliza el programa "Bambi lector" para la realización de actividades de práctica de la lectura y su posterior evaluación en sus clases de Lengua, del mismo modo A12, docente de Matemática y Formación Ética y Ciudadana, menciona la incorporación de Google Maps para: "ubicación en el plano, localización, posiciones de rectas en el plano/calles". A su vez, las entrevistadas de la institución B utilizan en sus clases libros en inglés de la biblioteca virtual, así como el programa Matepractic. En relación a este último, B9 explicó:

Es una plataforma digital, que lo que permite es trabajar con situaciones reales y concretas de la vida diaria, o sea son situaciones que le pueden pasar a los chicos y se hace todo manipulando la computadora. Les hace preguntas que implican un razonar, conocer y el aplicar, que son los tres puntos que se evalúan (B9).

Con este ejemplo se observa entonces que la aplicación de este programa implica la puesta en práctica y la evaluación de contenidos, pero, además, incluye el saber usar un recurso tecnológico.

Además, otra de las actividades que más se hizo presente en el discurso de las maestras fue el uso de tecnologías "como introducción al tema que se pretende desarrollar" (A14). Puntualmente en las entrevistas, A9 se refirió al uso de las TIC:

Me parece bien la incorporación y el uso. Me parece didáctico porque los chicos por ahí aprenden más escuchando algún vídeo o viendo el vídeo, mediante la imagen. (...) Es darle otro formato a la clase, a lo mejor, utilizarlo como partir para algún tema o mostrar algo que uno con la palabra no alcanza (A9).

También, como se mencionó, el 13\% de la muestra dijo aplicar las TIC en actividades vinculadas con la recreación educativa. Con esto, como se hizo mención en el marco metodológico, nos referimos a que las utilizan para escuchar música, sacar fotos, mirar películas, jugar a diferentes juegos con 0 sin un fin pedagógico o, simplemente, con el objetivo de generar un momento de recreación dentro de los contenidos escolares. Por ejemplo, A13 indicó que en sus actividades utilizaba: "Juegos de vocabularios y gramática (...) Cortos de dibujos animados", y a A1 cuenta que son los alumnos los que llevan sus pen drives para escuchar música durante determinados momentos del dictado de clase. Estas actividades también son mencionadas por A6 en su entrevista:

Entonces, en el televisor ponemos música de fondo mientras trabajan, a veces eligen ellos, si se pelean mucho elige uno, hemos visto películas, hemos usado lo grabadores comunes, ellos traen su pen y ponen música de fondo mientras trabajan, genera un clima muy lindo (A6).

Por otra parte, bien sabemos que para implementar determinada estrategia didáctica es necesario establecer previamente una planificación. Por esta razón, en los cuestionarios se les preguntó también por actividades con tecnologías planificadas a futuro. Como se observa a continuación en la figura 3 , el $18 \%$ de las docentes no respondió a este interrogante, no obstante, un $81 \%$ del total de las docentes planifican seguir incluyendo las tecnologías en sus prácticas. Este porcentaje estuvo distribuido entre aquellas que tienen pensado realizar las mismas actividades que ya mencionaron y aquellas que planifican otro uso, como por ejemplo: para presentar y ampliar temas, para el uso de Internet, para que los alumnos expongan trabajos, para la visualización de videos, para la búsqueda de información.

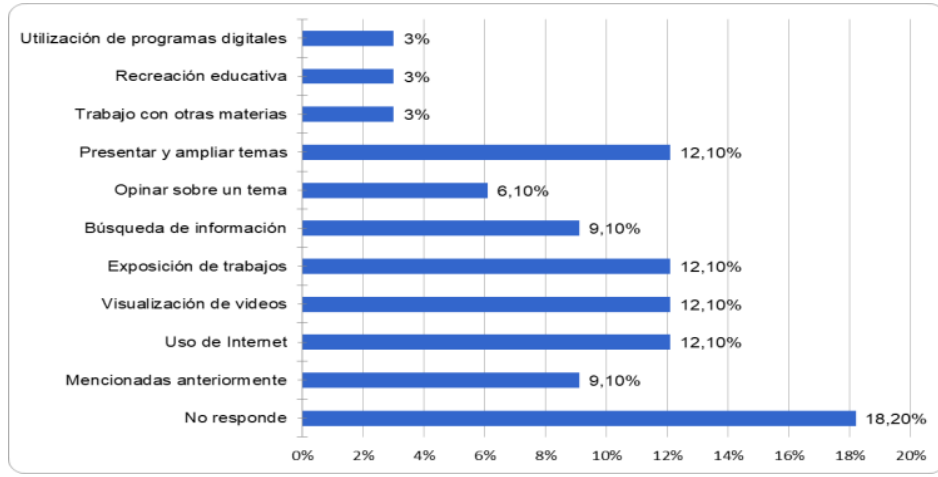

Figura 3. Futuras actividades a realizar con TIC 
Revista de la Escuela de Ciencias de la Educación, año 15, nRo. 14, vol. 2, Julio a Diciemre de 2019. Páginas 1-13. ISSN 1851-6297 (DESDE DICIEMBRE DE 2006 A DICIEMBRE DE 2017). ISSN 2362-3349 (EN LíNEA). LA INCORPORACIÓN DE LAS TIC EN LAS ESTRATEGIAS DIDÁCTICAS:

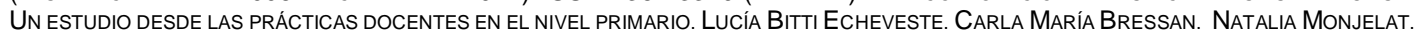

El porcentaje restante hizo mención a otras actividades como: la utilización de programas en general, el trabajo con otras materias, recreación educativa y actividades que impliquen opinar o debatir sobre algún tema.

Para complementar estos datos, se les consultó a los docentes en las entrevistas si los recursos tecnológicos disponibles en los contextos áulicos influían o no en la planificación y el desarrollo de sus clases. Al respecto, se destacaron palabras dichas por B2 y A9: "Si, lo tengo presente -a las tecnologías-, como algo a incluir en todas mis clases" (B2); "Sí, porque generalmente parto yo de algún... -recurso tecnológico- de ese uso. Cuando tengo que proyectar un vídeo, parto desde esa situación para después trabajar la clase" (A9). Las respuestas parecen indicar que las cuatro docentes coincidieron en que las TIC influyen en la planificación de sus clases. Adicionalmente, ambas docentes de la institución B afirmaron que planificar con tecnologías requiere invertir más tiempo y un trabajo extra. Por su parte, una de las maestras de la escuela A expresó que las tecnologías influyen en la planificación de sus clases porque se les abre el campo de acción o de trabajo y que "al cambiar los recursos de clase a clase, se hace más interesante, cuando uno es muy monótono con un recurso es aburrido para los chicos y para uno".

A su vez, como se ha mencionado, en las entrevistas además de indagar sobre los recursos que utilizan generalmente, se preguntó por los criterios en los que se basan a la hora de elegirlos.

Por un lado, las docentes de la institución A respondieron a la pregunta refiriendo específicamente a los recursos tecnológicos. Ambas, a su manera, coinciden en sostener que uno de los criterios en los que se basan para elegir los recursos es conocer, justamente, el uso de las TIC para su posterior aplicación durante las clases:

No toda tecnología suma, también hay que ver la manera en que uno la usa (...). Porque usarla no significa que sea ni para sumar, muchas veces se resta, si es un mal uso. Entonces hay que ser responsables en ese sentido, en la búsqueda y en la preparación" (A9).

Adicionalmente, la docente A6 dijo: "Primero, conocer el uso (...). Conocer la tecnología, cómo funciona y de qué manera, con anterioridad, (...) probarla y ver cómo funciona”.

Por otro lado, las maestras de la escuela B respondieron haciendo mención a los recursos en general, sin una mención específica a las TIC. Ambas docentes sostuvieron que previo a realizar la elección del recurso a utilizar, se debe considerar los objetivos que pretenden lograr. Por su parte, B2 relató:

Siempre teniendo en cuenta el objetivo de cada actividad y también la necesidad de cada grupo (...) es como que uno trata de buscar, bueno, el lugar y el recurso de acuerdo a ese objetivo que uno tenga que llegar (...) Pero siempre dependiendo de cada actividad, y no de cada clase, en realidad. Porque la clase, por lo menos en inglés, tiene millones de actividades y millones de momentos, con mucha dinámica distinta" (B2).

Y B9 agregó: "Primero tengo que tener en cuenta qué objetivo quiero trabajar para después ver qué contenido voy a trabajar para lograr ese objetivo (...) y pienso qué recurso es el que más me va a favorecer (B9).

Otro aspecto relevante que aparecía destacado en la revisión teórica es la capacitación. Por un lado, las profesionales de la institución B señalaron que habían recibido capacitaciones dentro de la institución, tanto sobre la modalidad de uso de las pizarras digitales interactivas, así como también capacitaciones relacionadas a programas específicos de sus materias. Al respecto, la docente B9 contó:

(...) me capacité yo, el colegio me hizo capacitar a mí a través de un skype con un chico de Logos, porque esta aplicación pertenece a Logos. Y después capacite al resto de las docentes (B9).

\section{La docente B2 agregó:}

De ciertas cosas, si recibimos capacitación. Por ejemplo, ahora nosotros en inglés estamos usando una plataforma virtual (...). Ahora, compraron esta licencia de esta plataforma entonces los chicos tienen una biblioteca virtual (B2).

Por su parte, las entrevistadas de la institución A, mencionaron no haber recibido capacitaciones específicas en tecnologías. En relación a esto, A6 expresó:

Sí, nos las mencionan -a las capacitaciones-... capacitaciones específicas no, por ahí uno sí puede consultar con compañeras que están dentro de las áreas (...). Desde la institución hay 
Revista de la Escuela de Ciencias de la Educación, año 15, nRo. 14, vol. 2, Julio a Diciemre de 2019. PÁGinas 1-13. ISSN 1851-6297 (DESDE DICIEMBRE DE 2006 A DICIEMBRE DE 2017). ISSN 2362-3349 (EN LÍNEA). LA INCORPORACIÓN DE LAS TIC EN LAS ESTRATEGIAS DIDÁCTICAS:

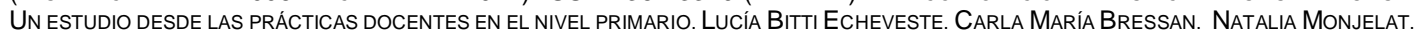

lineamientos generales, pero recién es como que estamos queriendo arrancar (A6).

\section{Discusión y conclusiones}

El análisis de los datos obtenidos permitió identificar las diferentes maneras de enseñar que tienen los docentes, ahondando puntualmente en los componentes y características de aquellas que incluían recursos tecnológicos. En cuanto a los componentes, fue posible identificar los recursos empleados en sus actividades, los criterios en los que se basan para seleccionarlos y la planificación educativa que establecen con los mismos. Además, los datos mostraron cuestiones vinculadas a las creencias de los participantes sobre las TIC, la disponibilidad tecnológica institucional y el valor de capacitación docente para el uso de las herramientas tecnológicas.

Aunque se observó que la totalidad de los participantes empleaban TIC, sus usos resultaron dispares en el marco de sus prácticas docentes, lo que condice con estudios previos que señalan que las estrategias dependen de la subjetividad, de los recursos existentes y del contexto en donde se llevan a cabo las acciones didácticas (Feo, 2010).

Los recursos tecnológicos más implementados fueron el TV smart, la computadora, el celular, las pizarras digitales interactivas y diferentes programas y plataformas digitales, aunque también surgieron tecnologías analógicas, observándose en general la incorporación de una amplia diversidad de recursos. Asimismo, esta diversidad está presente también en el tipo de actividades que se realizan con estos recursos tecnológicos, dentro de las cuales pudimos identificar: visualización de vídeos, recreación educativa, uso de programas digitales, presentación o ampliación de un tema, uso de Internet, exposición de trabajos por parte del alumnado, búsqueda de información, trabajo con otras materias o actividades que impliquen opinar o debatir sobre algún tema. En este sentido, pudimos observar que la mayoría de las docentes emplean las TIC para llevar a cabo las mismas actividades que plantean habitualmente, pero utilizando recursos tecnológicos.

Como se ha dicho, el disponer de infraestructura tecnológica y recursos informáticos es imprescindible (Garcés-Prettel, Ruiz-Cantillo y Martínez Ávila, 2014) pero en este estudio se obtuvieron datos que sugieren que en condiciones similares de disponibilidad tecnológica, una mayor integración puede estar relacionada con un mayor apoyo y sostén institucional de estas prácticas (Escontrela Mao y Stojanovic Casas, 2004), que se traduce por ejemplo en acciones formativas, como relataban las docentes de la institución B. Por ello, coincidimos con los planteos de Lerner (2012) y Cabello (2012) quienes señalaban que la incorporación de las TIC implica un proceso que se desarrolla con el tiempo, avanzando en la medida en que los actores institucionales lo conciban como una responsabilidad compartida, que requiere compromiso y dedicación de todos.

Por otra parte, sostenemos que para una correcta apropiación de las tecnologías es fundamental la formación no sólo en éstas, sino también en nuevas maneras de enseñanza. En este sentido, las docentes no se refirieron a las iniciativas provinciales o nacionales de formación (por ejemplo, el programa santafesino "Tramas Digitales"), sino que remitieron a capacitaciones a cargo de sus propias instituciones, y que parecen estar orientadas al uso instrumental de las tecnologías más que al uso pedagógico-didáctico. No obstante, si bien algunos autores referían a que hay docentes que consideran a las TIC como herramientas ajenas que terminan siendo un obstáculo para los objetivos pedagógicos o como causantes de temores porque ubican al alumno en una posición ventajosa, en esta investigación los datos no mostraron esto. Entonces, se podría concluir que la falta de formación para el uso de determinados recursos tecnológicos, no pareció ser un obstáculo para emplearlos en las clases (Garcés-Prettel et al., 2014) ya que todas las participantes indicaron su utilización de una u otra manera.

En suma, destacamos la importancia de haber estudiado las creencias y actitudes que tienen los docentes en relación con las TIC, ya que coincidimos con Roig (2016) en que es fundamental conocer cómo se percibe a las tecnologías y cómo se las utiliza. A partir de las entrevistas, podemos concluir que las docentes conciben a las TIC como herramientas que ayudan a que se den los procesos de enseñanza y aprendizaje. Las mismas, expresaron que las tecnologías son recursos que facilitan y mejoran la calidad de la enseñanza y la comprensión de un tema, lo cual lleva a que se genere un mayor interés en la clase por parte de los alumnos. A su vez, tanto en las entrevistas como en las encuestas se observó un uso de las TIC como soporte o complemento de las clases, sumándolas a los recursos que utilizan habitualmente para desarrollar los contenidos, coincidiendo con los señalados por investigaciones anteriores (Area Moreira, 2010).

Siguiendo con los ejes teóricos planteados, para implementar un material educativo y para aplicar determinada estrategia didáctica, es fundamental establecer una planificación educativa (Sánchez Rosal, 2010). En base a esto, observamos que la gran mayoría de las docentes dio a conocer su planificación, mencionando los recursos tecnológicos que seguirán utilizando y las actividades que continuarán realizando a futuro.

A modo de cierre y teniendo en cuenta los testimonios de los docentes, podemos concluir que las tecnologías digitales sí están siendo incluidas en las prácticas docentes. Esto es posible, en parte, gracias al 
Revista de la Escuela de Ciencias de la Educación, año 15, nRo. 14, vol. 2, Julio a Diciemre de 2019. Páginas 1-13. ISSN 1851-6297 (DESDE DICIEMBRE DE 2006 A DICIEMBRE DE 2017). ISSN 2362-3349 (EN LíNEA). LA INCORPORACIÓN DE LAS TIC EN LAS ESTRATEGIAS DIDÁCTICAS:

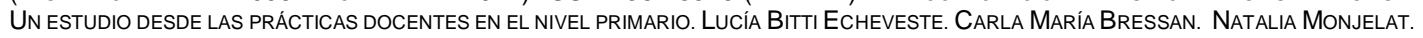

apoyo de los equipos directivos de las instituciones que pueden brindar los recursos necesarios para que esto suceda y además, al interés particular de los docentes por incluir estos recursos en sus estrategias didácticas.

Sin embargo, es necesario continuar el estudio de las diferentes características de las estrategias docentes cuando son mediadas tecnológicamente, realizando aportes al campo de la didáctica tecnológica (Litwin, 2005). En este sentido y a modo de sugerencia para futuras investigaciones, sería ideal llevar a cabo investigaciones que impliquen la aplicación de otros instrumentos de recolección de datos como las observaciones, complementando así los datos obtenidos de los discursos de las docentes, con la puesta en obra concreta dentro del aula. Para tal fin se debería profundizar en conocer el estilo de enseñanza, el tipo de estructura comunicativa que propone el docente para sus clases, el modo en que presenta los contenidos, la consigna que propone para acompañar dichos contenidos, entre otros.

Por otro lado, consideramos necesario destacar que, dado que la muestra proviene de dos instituciones educativas de la ciudad de Rosario, las conclusiones son válidas en relación a estas escuelas y de acuerdo a las respuestas dadas por las personas encuestadas y entrevistadas. En futuras investigaciones, sería interesante poder ampliar la muestra o trabajar con docentes de especialidades por ejemplo, para poder profundizar en el uso de las TIC. Asimismo, sería interesante abordar la problemática en instituciones de gestión pública o en diferentes niveles y modalidades del sistema educativo. Por otro lado, consideramos que sería favorecedor poder acceder a los documentos institucionales o trabajar con el equipo directivo de las instituciones para conocer en profundidad el lugar que ocupan las tecnologías dentro de la organización escolar.

\section{Referencias bibliográficas}

- $\quad$ Area Moreira, M. (2010). El proceso de integración y uso pedagógico de las TIC en los centros educativos. Un estudio de casos. Universidad de La Laguna. Tenerife, España: Facultad de Educación.

- Arnold, D., Spedding, A. y Pereira, R. (2013). Pautas metodológicas para investigaciones cualitativas y cuantitativas en ciencias sociales y humanas. Bolivia: UPIEB.

- Batista, M. A. (2007). Tecnologías de la información y la comunicación en la escuela: trazos, claves y oportunidades para su integración pedagógica. Buenos Aires: Ministerio de Educación, Ciencia y Tecnología de la Nación.

- $\quad$ Bixio, C. (2005). Las estrategias didácticas y el proceso de mediación. Enseñar a aprender. Rosario: Homo Sapiens.

- $\quad$ Burgess, J. R. D. y Russell, J. E. A. (2003). The effectiveness of distance learning initiatives in organizations. Journal of Vocational Behavior, 63, pp.289-303.

- Cabello, R. (2012). Palos en la rueda. Cinco factores de resistencia a la integración de Internet en la escuela. En D. Goldin; M. Kriscautzky y F. Perelman (Eds.), Las TIC en la escuela, nuevas herramientas para viejos y nuevos problemas, pp.183-216. España: Editorial Océano Travesía

- Cabello, R. y Levis, D. (2007). Medios Informáticos en la Educación Introducción a principio de siglo XXI. Buenos Aires: Prometeo Libros.

- Canales, F. (2017). Criterios de uso de las tecnologías de la información y comunicación (TIC) como la computadora y el teléfono móvil por parte de docentes y alumnos de una escuela nueva de la ciudad de San Lorenzo (Tesis de Licenciatura en Psicopedagogía). Rosario, Argentina: Universidad Nacional de General San Martín.

- Cariaga, R. (2018). Experiencias en el uso de las TIC. Análisis de relatos de docentes. Ciencia, 29(56), pp. 131-155.

- $\quad$ Cid-Sabucedo, A., Pérez-Abellás, A. y Zabalza, M. A. (2009). Las prácticas de enseñanza declaradas de los "Mejores Profesores" de la Universidad de Vigo. Relieve, 15 (2), pp. 1-29.

- Cole, M. (1984). La zona de desarrollo próximo: donde cultura y conocimiento se generan mutuamente. Infancia y Aprendizaje, 25, pp. 3-17.

- Coll, C. (2009). Aprender y enseñar con las TIC: expectativas, realidades y potencialidades. En R. Carneiro, J.C. Toscano y T. Díaz (Eds.) Los desafíos de las TIC para el cambio educativo (pp.113-126). Buenos Aires: Santillana.

- $\quad$ Di Paolo, B. (2014). La experiencia de las Tecnologías de la Información y la Comunicación (TICs) en el aula: disciplina, control, no-políticas. FHyCS-UNJu, 45, pp. 11-25.

- Dussel, I. y Quevedo, L. A. (2010). Educación y nuevas tecnologías los desafíos pedagógicos ante el mundo digital. Buenos Aires: Santillana.

- Escontrela Mao, R. y Stojanovic Casas, L. (2004). La integración de las TIC en la educación: Apuntes para un modelo pedagógico pertinente. Revista de Pedagogía, 25(74), pp. 481-502.

- Feo, R. (2010). Orientaciones básicas para el diseño de estrategias didácticas. Tendencias Pedagógicas, 16 (16), pp. 221-236.

- Garcés-Prettel, M., Ruiz-Cantillo, R. y Martínez Ávila, D. (2014). Transformación pedagógica mediada por tecnologías de la información y la comunicación (TIC). Revista Saber, Ciencia y Libertad, 9(2), pp. 217-228. 
Revista de la Escuela de Ciencias de la Educación, año 15, nRo. 14, vol. 2, Julio a Diciemre de 2019. Páginas 1-13. ISSN 1851-6297 (DESDE DICIEMBRE DE 2006 A DICIEMBRE DE 2017). ISSN 2362-3349 (EN LíNEA). LA INCORPORACIÓN DE LAS TIC EN LAS ESTRATEGIAS DIDÁCTICAS: Un EStUdio desde las prácticAs docentes en el nivel primario. Lucía BitTI ECheVeste. Carla María Bressan. Natalia Monjelat.

- González, M. G. y Ramírez, I. R. (2010). Enseñar a aprender, un reto para la formación de profesionales universitarios en el nuevo siglo. ODICEO, Revista Electrónica de Pedagógía, 7 (14), pp. 1.

- González Jaimes, E. I., Hernández Prieto, M. de L., Zea, J. M., Palmer, J. A., Hand, D., Mannila, H., Velasco M. y Mosquera F. (2015). Estrategias didácticas para el Aprendizaje Colaborativo. Igarss 2014. 29 (2), pp. 1-5.

- Guiller, C. (2015). Prácticas de enseñanza con TIC en la escuela primaria (Proyecto de investigación). Instituto Superior de Formación Docente $\mathrm{N}^{\circ} 17$, La Plata.

- Hernández Sampieri, R., Fernández Collado, C. y Baptista Lucio, M. (2014). Metodología de la investigación. México: McGraw-Hill.

- Lerner, D. (2012). La incorporación de las TIC en el aula. Un desafío para las prácticas escolares de lectura y escritura. En D. Goldin; M. Kriscautzky y F. Perelman (Eds.), Las TIC en la escuela, nuevas herramientas para viejos y nuevos problemas, pp.23-88. España: Editorial Océano Travesía.

- $\quad$ Litwin, E. (2005). Tecnologías en tiempos de Internet. Buenos Aires: Amorrortu.

- $\quad$ Lugo, M. T. y Kelly, V. (2011). La matriz TIC. Una herramienta para planificar las tecnologías de la información y la comunicación en las instituciones educativas. Ciudad Autónoma de Buenos Aires: Instituto Internacional de Planeamiento de la Educación IIPE-Unesco.

- Miranda, M. P. (2013). Las Nuevas Tecnologías de la Información y la Comunicación en aulas primarias rosarinas (Tesis de Licenciatura en Psicopedagogía). Rosario, Argentina: Universidad Nacional de General San Martín.

- $\quad$ Rivero Cárdenas, I., Gómez Zermeño, M., Fernando, R., y Abrego Tijerina, A. (2013). Tecnologías educativas y estrategias didácticas: criterios de selección. Revista Educación y Tecnología, 3, pp. 190-206.

- Roig, H. (2016). Tecnologías en la enseñanza: ¿introducirlas o percibirlas? Novedades Educativas, 300-301, pp.56-60.

- Sabino, C. (1996). El proceso de investigación. Buenos Aires: Lumen-Humánitas.

- Salvucci Gaido, A. (2017). El uso de las TIC en la transmisión de contenido curricular por medio de las aulas digitales móviles, por parte de docentes de segundo ciclo de una escuela pública del sur de Santa Fe (Tesis de Licenciatura en Psicopedagogía). Rosario, Argentina: Universidad Nacional de General San Martín.

- Sánchez Rosal, A. (2010). Estrategias didácticas para el aprendizaje de los contenidos de trigonometría empleando las TICS. Revista Electrónica de Tecnología Educativa, 31, pp. 1-19.

- Tedesco, J. C.; Steinberg, C. \& Tófalo, A. (2015). La integración de TIC en la educación básica en Argentina. Buenos Aires: Unicef.

- Tófalo, A. (2015). Las Tic y la educación primaria en la Argentina. Programa TIC y Educación Básica en la Argentina. Buenos Aires.

- Tófalo, A. (2017). Informe: Aprender 2016: Acceso y uso de TIC en estudiantes y docentes. Buenos Aires, Argentina: Secretaría de Evaluación Educativa del Ministerio de Educación de la Nación. 